\title{
Optimizing IEEE 802.16j: Multihop Relaying in WiMAX Networks
}

\author{
${ }^{1}$ Sapna Parmar, ${ }^{2}$ Priyanka Dhasal \\ Computer Science \& Engineering \\ Patel Collage of Science and Technology, Indore, MP, India
}

\begin{abstract}
For the global deployment of mobile WiMAX networks the limited budget of installation and efficiency of network quality are very important concerns. To optimize the WiMAX network quality of services in term of good data rate guarantee and the efficient radio spectrum to serve as more as possible users more and more coverage should be provided. For enhancing the radio coverage of the WiMAX network multihop relay station technique is proposed. IEEE 802.16 forum gives different types of service flows (i.e. UGS, rtps, ertps, nrtps, BE) for various kinds of applications. The network coverage can be improved by properly locating the relay stations to optimal locations where the installation of base station could be more costly as compared to relay stations. In this paper we presented the overview of IEEE 802.16j multihop relay networks with QoS support and how the network coverage and quality will be improved for rural and hilly areas where installation of base station is a big challenge.
\end{abstract}

\section{General Terms}

QoS Scheduling Services UGS, rtPS, ertPS, nrtPS, BE, Relay Station, Network Simulator.

\section{Keywords}

IEEE802.16j, MIMO, OFDMA.

\section{INTRODUCTION}

We In order to be able to deliver omnipresent widespread services for the next generation of wireless technology like WiMAX, LTE or other 4G technologies, it has become mandatory in recent years that, the network must provide excellent coverage, and obviously improved bandwidth per subscriber station [1]. In order to achieve that at frequencies above 2 and $3 \mathrm{GHz}$, which are targeted for future wireless technologies, network architecture must reduce significantly the cell size or the distance between the network and subscribers' antennas One solution introduced with the WiMAX 802.16j [2] standard is the wireless Multi-hop Relay Station (MRS), intended to overcome these challenges. On one hand, it should be small, cost-effective and easy to install for enabling mass deployment in indoor and outdoor environments and creating relatively small areas with excellent coverage and high capacity availability. On the other hand, it does not require any dedicated backhaul equipment as it receives its capacity from centralized base-stations via the same resources used for the access service. In a setting where a MRS exists, enabling MIMO transmission [3], the link referred to needs to be specified. This means that, when a $2 \times 2$ setting is mentioned, there can be either two transmit antennas on the base station and two receive antennas on the relay station, or two transmit antennas on the relay station and two receive antennas on the subscriber's device [3].Wireless Multi-hop Relay Stations (MRSs), when deployed in various sights, result in increased throughput or coverage as shown in Figure 1. A general case, where a relay station can be used, is in situations with coverage constraints such as areas where there is presence of physical obstacles (e.g. buildings, forests), or in indoor coverage cases. Some examples are large office buildings, University campuses, and villages in unreachable areas on rockier uplands etc. Another scenario, where MRSs can be used, is for high mobility users with increased bandwidth requirements, such as trains with a great number of wireless users. Such a mobile subscriber will more likely have data rate degradations due to non-fixed position. In this case, a relay station can be considered as the most feasible solution in terms of cost and coverage. 


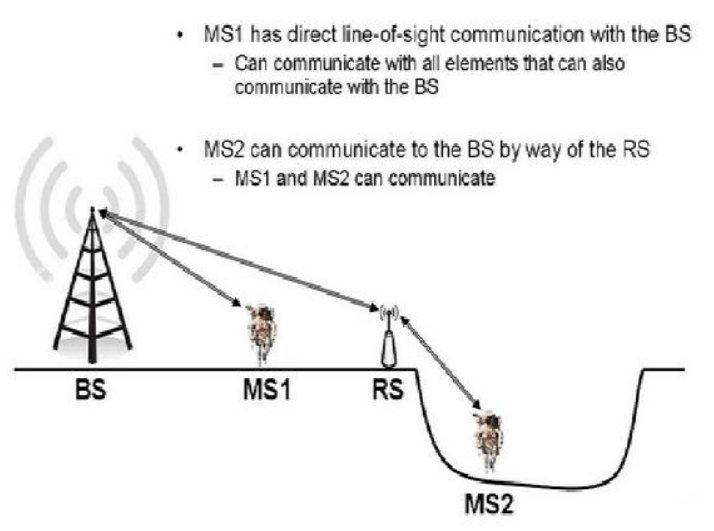

Fig 1: Extending Coverage by Using Relay Station in WiMAX Network

\section{IEEE 802.16j TECHNOLOGY}

The essentially core element of IEEE802.16j standard for air interface are - the PHY (physical) layer and the MAC (Media Access Control) layer [4]-[5]. This section provides an overview of the technology employed in these two layers in the mobile $802.16 \mathrm{j}$ specification.

\subsection{PHY Layer}

As a carrier of various data $802.16 \mathrm{j}$ uses scalable OFDMA, supporting channel bandwidths of between $1.25 \mathrm{MHz}$ and $20 \mathrm{MHz}$, with up to 2048 sub-carriers [6]. It supports adaptive modulation and coding, so that in conditions of good signal, a highly efficient 64 QAM coding scheme is used, whereas when the signal is poorer, a more robust BPSK coding mechanism is used. In intermediate conditions, 16 QAM and QPSK can also be employed. Other PHY features include support for Multiple-in Multiple-out (MIMO) antennas in order to provide good non-line-of-sight propagation (NLOS) characteristics (or higher bandwidth) and Hybrid automatic repeat request (HARQ) for good error correction performance.

Although the standards allow operation in any band from 2 to $66 \mathrm{GHz}$, mobile operation is best in the lower bands which are also the most crowded, and therefore most expensive.

\subsection{MAC Layer}

Depending on how wire line technologies such as Ethernet, Asynchronous Transfer Mode (ATM) and Internet Protocol (IP) are encapsulated on the air interface, and how data is classified, etc, the $802.16 \mathrm{j}$ MAC describes a number of Convergence Sub layers [7]. It also describes how secure communications are delivered, by using secure key exchange during authentication, and encryption using Advanced Encryption Standard (AES) or Data Encryption Standard (DES) during data transfer [8]. Further features of the MAC layer include power saving mechanisms (using Sleep Mode and Idle Mode) and handover mechanisms.

A key feature of $802.16 \mathrm{j}$ is that it is a connection oriented technology. The subscriber station (SS) cannot transmit data via Relay Station (RS) until it has been allocated a channel by the Base Station (BS). This allows $802.16 \mathrm{j}$ to provide strong support for Quality of Service (QoS).

\section{QOS OVER IEEE 802.16j}

When we are talking about air interface, maximum tolerable latency and jitter are the 
major QoS parameters defined by IEEE 802.16 WiMAX network. When the wireless network with QoS mechanisms combined over the wired backbone, it must provide predictable end-to-end QoS [9]-[10]. When Multi-hop Relay is introduced, QoS must be applied to two wireless links - one is the link between the MS and the RS, and the other is the link between the RS and the BS. It has become a big problem of assigning Service Flows for individual QoS parameters for each of the wireless links. In MAC-based relay network, this can be done by continuously monitoring the CID in each PDU and associating each CID or SF with QoS parameters over both the link. For relay based network, the CID is not available but the service flow (SF) can be deduced from the GRE (Generic Routing Encapsulation) tunnel used to transport the data path. In this sense, relay-based MAC and network-based MAC provide equivalent end to end QoS. 802.16j MAC-based relay also defines Tunnel CIDs. A Tunnel-CID is basically a connection which is used to traverse every intermediate hops between a RS and the BS. Several user-facing Service Flows are aggregated into only one Tunnel-CID, and this only Tunnel-CID has a set different QoS parameters. It is the base station who schedules the resources for the Tunnel-CID and if it is s a single connection with given QoS parameters, it ignores the other Service Flows. By aggregating Service Flows into a Tunnel-CID we can simplifies scheduling and it also can reduce bandwidth requirements.

Quality of service (QoS) in IEEE 802.16j is supported by allocating each connection between the SS and the BS (called a service flow in IEEE 802.16 terminologies) to a specific QoS class [11]. The BS and the SS use a service flow with an appropriate QoS class (plus other parameters, such as bandwidth and delay) to ensure that application data receives QoS treatment appropriate to the application. IEEE 802.16 supports five different QoS scheduling which will be described next.

\subsection{QoS Scheduling}

Please IEEE 802.16 WiMAX MAC layer support five different scheduling services; using these scheduling services, WiMAX is enabling to offers the different applications varying size of bandwidth associated with the priority of the application, in comparison to Wi-Fi where each application has the same priority. It is the BS scheduler that distributes the amount of bandwidth necessary for an application, depending on which QoS class the application has. With the QoS parameters described next, the BS can offer polls and or grants at adequate occasions. Following are the five scheduling service types [12]:

1. UGS-This is Unsolicited Grant Service generally used for real-time applications, Within that the BS grants fixed-sized data packets, at periodic intervals, Thus, this type of scheduling is suitable for real-time data streams, example includes VoIP.

2. rtPS- This is Real-Time Polling Service and has the same functionality as UGS have, but instead of granting fixed-sized data packets, ertPS uses variable size data packet distribution. Example includes video streaming, i.e. MPEG (Moving Pictures Experts Group).

3. ertPS- This is Extended Real-Time Polling Service This scheduling service type is based on both UGS and rtPS. It is adequate for applications as VoIP, in which the data rate is variable. The typical example includes video conferencing by using Skype.

4. nrtPS- The Non-Real-Time Polling Service requires minimum data rates for data packets with variable size. It is made with the intention of managing delay and is therefore convenient in the use of File Transfer Protocol (FTP). The BS regularly offers request polls, which makes it possible for the application to make requests even though network congestion is in progress.

5. BE- The Best Effort scheduling mechanism can cause long delays when there is network congestion since it handles applications on best available basis. It has no support for applications which have requirements of 
minimum service guarantees. An example of an application that uses this scheduling service is E-mail. [11]

\subsection{QoS Parameters}

The The qualities of services usually vary application to application as requested and depend on the following parameters [13]:

-Bandwidth

-Latency or Delay

-Jitter or Variation in delay

-Synchronization in between multiple streams (e.g. the voice should be synchronized with the video)

-Packet or Cell loss ratio (\% of packet/cell dropped)

-Cell/Packet error ratio (\% of packet/cells arriving with one or more errors)

\section{PREFERRED TOOL: NETWORK SIMULATOR}

The network simulator is originally developed for network research work. Network simulator is only using the languages $\mathrm{OTcl}$ and $\mathrm{C}++$. $\mathrm{C}++$ is preferred for overall design of protocols because it is easy to run. Also, any kind of settings change using $\mathrm{C}++$ would be the slow process that's why OTcl is to prefer [16], where changes can be made more interactively. Building the actual network virtually and analysis of that network with great efficiency before deployment is a tedious job, for this simulation work we will prefer the ns-3 simulator [14] together with the NIST WiMAX module [15].

A network simulator is a piece of software or hardware that predicts the behavior of a network, without an actual network being present. A network simulator is a software program that imitates the working of a computer network. In simulators, the computer network is typically modeled with devices, traffic etc. and the performance is analyzed.
Network simulators serve a variety of needs. Compared to the cost and time involved in setting up an entire test bed containing multiple networked computers, routers and data links, network simulators are relatively fast and inexpensive. They allow engineers, researchers to test scenarios that might be particularly difficult or expensive to emulate using real hardware - for instance, simulating a scenario with several nodes or experimenting with a new protocol in the network. Network simulators are particularly useful in allowing researchers to test new networking

\subsection{NS Design}

NS-3 is primarily useful for simulating local and wide area networks. NS is Object-oriented $\mathrm{Tcl}(\mathrm{OTcl})$ script interpreter that has a simulation event scheduler and network component object libraries and network setup module. The ns-3 library is wrapped to python thanks to the pybindgen library which delegates the parsing of the ns-3 C++ headers to gccxml and pygccxml to generate automatically the corresponding $\mathrm{C}++$ binding glue [17].

The large majority of its users focus on wireless simulations which involve models for Wi-Fi, WiMAX, or LTE for layers 1 and 2 and routing protocols such as OLSR and AODV These automatically-generated $\mathrm{C}++$ files are finally compiled into the ns-3 python module to allow users to interact with the $\mathrm{C}++\mathrm{ns}-3$ models and core through python scripts.

\section{CONCLUSION AND FUTURE WORK}

Building As relays can permit a faster network rollout and thus because of simplicity, flexibility, deployment efficiency and cost effectiveness, relay technology has focused significant attention. NIST aiming to develop an effective relay station implementation for WiMAX technology as a demanding $4 \mathrm{G}$ 
technology. We have discussed several architecture principles and benefits for relaybased networks and we have presented an essential description of the related RS software architecture design (PHY and MAC layers architecture) for enabling QoS in IEEE 802.16j WiMAX Network. We basically are focusing in the core concept of multi-hop relay network architecture, adopting QoS in the IEEE 802.16j standard.

In this article we provides an overview of IEEE802.16j technology, focusing on some of the most pertinent aspects related to cost optimization and also we provide the overview of the tool used for the simulation work that is network simulator. By using the less expensive relay stations, the radio network will definitely be increased, but the efficiency of network may be affected for different types of user demanding scheduling services. For the future work we can evaluate the performance of QoS with different parameters in IEEE 802.16j WiMAX network.

\section{ACKNOWLEDGMENTS}

We would like to thank our peer reviewers to review the paper and giving constructive suggestions in the right direction for completion of this paper.

\section{REFERENCES}

[1] Mark Norris and Adrian Golds. Why WiMAX? URL: http://www.intercai.co.uk/library/pdf/Why-WiMAX.pdf.

[2] Steven W. Peters et al "IEEE The Future of WiMAX: Multihop Relaying with IEEE 802.16j," IEEE Communications Magazine • January 2009, pp. 2-9

[3] B. Muquet et al "MIMO Techniques for Mobile WiMAX Systems," SEQUANS Communications, 2009

[4] IEEE Std 802.16j тм -2009 (Amendment to IEEE Std. 802.16-2009) IEEE Standard for Local and metropolitan area networks Part 16: Air Interface for Broadband Wireless Access Systems IEEE 3ParkAvenue New York, NY 10016-5997, USA, 12 june 2009

[5] EEE 802.16-2005: IEEE Standard for Local and Me tropolitan Area Networks - Part 16: Air Interface for Fixed and Mobile Broadband Wirele ss Access Systems - Amendment 2: Physical Layer and Medium Access Control Layers for Comb ined Fixed and Mobile Operation in Licensed Bands, February 2006
[6] Hassan Yaghoobi et al" Scalable OFDMA Physical Layer in IEEE 802.16 WirelessMAN," (2004)

[7] Toumpakaris, D. et al" A survey on next generation mobile WiMAX networks: objectives, features and technical challenges," Communications Surveys \& Tutorials, IEEE (Volume:11 , Issue: 4 ), Fourth Quarter 2009, pp-3-18.

[8] Johnston, D. et al" Overview of IEEE 802.16 security," Security \& Privacy, IEEE (Volume:2, Issue: 3 ), MayJune 2004,pp-40-48.

[9] K. Etemad"Overview of Mobile WiMAX Technology and Evolution", IEEE Communication Magazine., vol. 46, no. 10, pp.245 -290.

[10] Talwalkar, R.A. et al" Analysis of Quality of Service (QoS) in WiMAX networks," Networks, 2008. ICON 2008. 16th IEEE International Conference on ,12-14 Dec. 2008, pp. 1 - 8

[11] Belghith, A. et al "Comparison of WiMAX scheduling algorithms and proposals for the rtPS QoS class," Wireless Conference, 2008. EW 2008. 14th European ,22-25 June 2008,pp. 1 - 6

[12] http://www.tranzeo.com/allowed/Tranzeo_WiMAX_QoS _Classes_Whitepaper.pdf

[13] Chakchai So-In et al" Scheduling in IEEE 802.16e mobile WiMAX networks: key issues and a survey," Selected Areas in Communications, IEEE Journal on (Volume:27, Issue: 2 ) February 2009,pp-156 - 171

[14] Ns-2. URL: http://www.isi.edu/nsnam/ns/.

[15] Simulation Models for NS-2. URL: http://www.nist.gov/ itl/antd/emntg/ssm tools.cfm.

[16] Loutfi Nuaymi Aymen Belghith. Design and Implementation of a QoS-included WiMAX Module for NS-2 Simulator. Tech. rep. TELECOM Bretagne, 2008

[17] M. Lacage and T. R. Henderson. Yet another network simulator. In WNS2 '06: Proceeding from the 2006 workshop on ns-2: the IP network simulator, page 12, New York, NY, USA, 2006. ACM. 